\title{
From the neo-Kraepelinian framework to the new mechanical philosophy of psychiatry: regaining common sense
}

\begin{abstract}
Ridha Joober, MD, PhD; Karim Tabbane, MD
"While the other senses put us in contact with things, the common sense presides over our relations with persons."

Henri Bergson

The neo-Kraepelin framework (NKF) dominated psychiatric practice and research for almost 50 years. In this editorial, we briefly review the historical context in which the NKF emerged and reflect on its current implications for clinical

lection, the European masters of psychiatry contributed to the invention of various theoretical frameworks, in which masterfully described cases were taken as exemplars to support specific models of psychopathology and were used to understand the life histories of patients, the pathways leading from normal to pathological behaviours, the grouping of patients into diagnostic categories and the treatment offered according to the models at hand.
\end{abstract} practice and research. We conclude with some reflections on how this framework is adapting to the mechanical philosophy of biology that emerged at the turn of the 21st century.

The practice of psychiatry in the 18th and 19th centuries produced a rich literature in the form of detailed clinical descriptions. These efforts were made with the premise that systematic observations are the basic method for advancing sciences, including the nascent science of behavioural medicine. Philippe Pinel (1745-1826), one of the founding fathers of psychiatry, wrote in his Treatise On Insanity that the path to success required him "to notice successively every fact, without any other object than that of collecting materials for future use; and to endeavour, as far as possible, to divest myself of the influence, both of my own prepossessions and the authority of others." ${ }^{11}$ Given the wide range of human behaviours, emotions and cognition, as well as their quasiinfinite variations and combination from one subject to the other and the limitless ways an observer could interact with and interpret the behaviour of their patients, there were no boundaries to what could be reported and discussed.

The aspiration to divest influence from oneself and that of authority is laudable; however, it has been strongly criticized and is now considered epistemologically flawed, particularly in human sciences. Indeed, all observations are read/ decoded according to various theoretical frameworks, or disciplinary matrices, ${ }^{2}$ that are required for all levels of analyses, starting from the simplest descriptions to the highest levels of causal or other organizing principles. Thus, beyond fact col-

\section{Nineteenth-century frameworks of psychiatric disorders}

Although it is difficult to do justice to the large number of authors who have marked the history of psychiatry, 2 major frameworks, 1 proposed by Emile Kraepelin and 1 by Sigmund Freud, were highly influential and shaped modern psychiatry significantly. A brief presentation of these 2 frameworks here will serve to situate them historically and underline their effects on the emergence of the NKF.

Emile Kraepelin (1856-1926) is probably the most consequential researcher for modern psychiatry. ${ }^{3}$ Marked by the European tradition of correlating signs and symptoms and tracking their evolution over time to define diseases, as typified by general paresis and its association with a specific pathogen, his main legacy to psychiatry has been the distinction between dementia præcox and manic-depressive illness as 2 different illnesses with putatively different pathological processes, genetics and biochemistry. ${ }^{4}$ His approach is the closest to the medical model. The Kraepelinian system was quickly adopted by the American Psychiatric Association, and it replaced previous classification systems based mainly on symptoms. ${ }^{5}$

Sigmund Freud (1856-1939), the founder of psychoanalysis, arguably the most influential and popular theoretical framework of the mind and its workings in the 20th century, did not seek to construct a psychiatric nosography, but rather

Correspondence to: R. Joober, Douglas Hospital Research Centre of Psychiatry, 6875 Blvd Lasalle, Montreal, Que., H4H 1R3; ridha.joober@mcgill.ca

DOI: $10.1503 / j p n .180240$ 
elaborated a theory of the psychic apparatus. He postulated 3 fundamental agencies - the id, ego and superego - and emphasized a major role of the id (unconscious) in human behaviours. These agencies are subject to conflicts between them and the outside world, leading to the emergence of symptoms, which are conceived as maladaptive defence mechanisms. Over time, psychoanalytical theories have burgeoned, seeking to explain all kinds of signs and symptoms using the initial psychic topology of Freud and all their avatars. Although its impact on human sciences in general and psychiatry in particular was major and universal, psychoanalysis was severely criticized by some philosophers of science as being pseudoscientific. ${ }^{6}$

\section{The neo-Kraepelinian framework}

While the Kraepelinian and other 19th-century frameworks of psychiatric disorders continued to thrive along with psychoanalysis in Europe, American psychiatry was almost completely engulfed by the theories of Freud and his disciples in the '60s and '70s. ${ }^{7,8}$ This resulted in a major rift between American psychiatry and mainstream European psychiatry. The climax of this rift was epitomized by the realization that clinicians and researchers in Britain and the United States were using the same label to designate highly disparate disorders. ${ }^{9,10}$ For example, it was found that the designation of schizophrenia in the United States included depressive, manic and personality disorders. In response to this appalling state of affairs, major efforts were deployed to improve the reliability of the designation of psychiatric disorders, mainly in the form of creating operationalized criteria to diagnose mental disorders, which were included in the Diagnostic and Statistical Manual of Mental Disorders, third edition (DSM-III), published by the American Psychiatric Association in 1980. This work was led by a group of American psychiatrists called the neo-Kraepelinians, and it received immediate, wide acceptance and usage all over the world. The major premise of the work was that a homogenization of the language used by clinicians and researchers was required for any serious attempt to develop a scientific understanding of psychiatric disorders. An important aspect of this effort to homogenize language was the reliance on criteria that had high interrater reliability. Another major theoretical foundation of the NKF was the repudiation of any top-down theoretical framework of mental illnesses. It was assumed that using reliable criteria to define disorders could, through an iterative process of validation, lead to bottom-up theories of mental illnesses.

Despite widespread acceptance of the NKF, it is important to review some of its consequences and reflect on its standing in view of the major developments in biological psychiatry, notably genetics and, to a lesser extent, brain imaging.

In the pursuit of reliability, major efforts were made to exclude any signs and symptoms with poor interrater reliability. Consequently, many of these signs and symptoms that were important - even foundational for the definition of some psychiatric disorders - were disregarded. One well-known example, that of psychotic ambivalence, was considered a car- dinal sign of schizophrenia, as conceived by Bleuler, but was not included in the DSM-III and its subsequent versions because it did not fulfill the criterion of satisfactory interrater reliability. However, ambivalence is certainly a clinical reality in some patients that can be clearly perceived by phenomenologically tuned clinicians and can possibly be very helpful to better understand the patient and relate to their difficulties. Another symptom, delusional mood, which had a pre-eminent place in the understanding of the early phases of psychotic illness, has almost disappeared in the modern psychiatric literature. This experience of delusional mood was one of the fundamental insights into the phenomenological experience of individuals facing the early dissolution of the structure of consciousness as a consequence of the psychotic process. Other symptoms, such as delusions, although pre-eminent in the DSM system, are restricted to a bare definition of false beliefs with some modifiers, which contrasts with the sophisticated phenomenological analyses offered by some masters of psychopathology who consider delusion a "particular type of existence in the world, which should be analyzed on a case-bycase basis and not defined in advance by its nonreality."11,12,13

Here, we have presented a few examples of how listoriented psychiatry has resulted in the loss of a rich clinical and semeiotic knowledge painstakingly identified by psychiatrists and researchers over the last 2 centuries. The purpose at this point is not to blame the successive versions of the DSM for this impoverishment of clinical psychiatry. Indeed, the NKF never purported the DSM to be a clinical textbook to teach psychiatry and improve empathy. Nonetheless, an unintended consequence of the NKF, unfortunately, is the "death of phenomenology" in America, as Nancy Andreasen argued a few years ago. ${ }^{1}$

Notwithstanding the unintended negative consequences of criteria-oriented psychiatry, we believe that this approach made a major contribution to psychiatric research, because patients could be grouped under relatively reliable labels and studied with modern and tremendously sophisticated technology that transformed all aspects of medicine (e.g., molecular biology, genetics, high-resolution brain imaging, highthroughput computational power). Had we continued to operate with the confused language that predated the NKF, our field would have developed into a deafening cacophony. This approach enabled researchers in psychiatry to apply cutting-edge technologies developed in the last 40 years in an attempt to validate these criteria-defined disorders (this is what most papers published in JPN attempt to do). These efforts represent the second and major part of the NKF agenda. Robins and Guze, who were major figures among the neo-Kraepelinians, published the "manifesto" of this validation program in $1970 .{ }^{14}$ They proposed 5 criteria - namely, clinical description, laboratory study, exclusion of other disorders, follow-up study and family study — to validate psychiatric disorders. They gave the example of how family study led them to conclude, "good prognosis 'schizophrenia' is not mild schizophrenia, but a different illness." We believe that given the tremendous developments in genetic and brain imaging research, it is legitimate to reflect on whether we have gained any added validity, as proposed by Rubins and 
Guze, of any specific major psychiatric disorder compared with our basic clinical knowledge. Most of the examples that follow pertain to schizophrenia, but the conclusions can be extended, without loss of generality, to all mental disorders.

The publication of major gene effects associated with schizophrenia ${ }^{15}$ and bipolar disorder ${ }^{16}$ in the late ' 80 s was met initially with enthusiasm, only to be refuted extensively later on. It can now be affirmed without any doubt that there are no major mutations that are causative of any mental disorder, although a handful of cases may be strongly influenced by mutations in a single gene (e.g., complement C4, RNA-motif binding 12, and SETD1A in schizophrenia). Furthermore, the picture that emerged from the very large and statistically powerful international collaborative effort (Psychiatric Genetic Consortium; PGC) that assembled tens of thousands of samples of patients and controls and compared their genetic variants is quite sobering from the perspective of the NKF. The most recent genome-wide association study (GWAS) in 36180 Chinese participants, along with a concomitant analysis of the PGC data, identified 113 single nucleotide polymorphisms (SNPs) that were significantly associated with schizophrenia, ${ }^{17}$ and each of these variants had a tiny effect. Genome-wide association studies have also developed and validated the concept of a polygenic risk score, which reflects the aggregate effect of thousands of loci that might be implicated in a disorder under a polygenic model. This work has clearly demonstrated a highly significant genetic overlap among most of the major psychiatric disorders that were included in the analysis. ${ }^{18-21}$ Most remarkably, a recent, large study (265218 patients and 784643 controls) investigated the genetic commonalities among 25 brain disorders. The study found that psychiatric disorders and neurologic disorders generally do not share genetic variants, establishing a clear boundary between psychiatric and other disorders of the brain. In addition, the study found that, contrary to neurologic disorders, which show clear boundaries among themselves, psychiatric disorders do not. For example, schizophrenia has significant genetic correlation with attention-deficit/ hyperactivity disorder (ADHD), anorexia nervosa, bipolar disorder, autism and major depressive disorder. ${ }^{22}$ These nonspecific genetic influences of SNPs on a large number of psychiatric disorders have also been shown in relation to genomic copy number variants (CNVs) that have shown stronger genetic effects, but also poor specificity. ${ }^{23}$ Indeed, it is now believed that any of these given pathogenic CNVs increase the risk for neurodevelopmental abnormalities and a host of psychiatric outcomes (e.g., ADHD, autism, intellectual disability, learning disabilities, epilepsy).

Genetic molecular studies have also highlighted a few important facts that have clear implications for our understanding of the architecture of psychiatric disorders. It has been reported that more than $71 \%$ of $1 \mathrm{MB}$ windows in the human genome contain 1 or more genetic variants, which increase the risk for schizophrenia, ${ }^{24}$ and the majority of these variants are noncoding variants with weak enrichment in functional gene categories. These observations, which are shared by complex human disorders, led to the formulation of the "omnigenic" model, postulating that the disease risk is mainly due to genetic variants with no specificity to any particular disorder; however, they convey their effects through widely distributed pleiotropic effects on transcription. But how a specific disorder emerges from these largely distributed effects is far from being understood..$^{25}$

Thus, the genetic architecture of the major psychiatric disorders did not support the well-delimited entities reified as diseases through highly reliable criteria. Instead, molecular genetic studies support that the major mental disorders cluster in a large group, with very fuzzy delimitation among the different disorders. Consequently, under the highly polygenic nature and the complex genetic architecture of psychiatric disorders, it can be safely asserted that even affected members in the same family will differ from each other with regard to thousands of genetic variants implicated in the disorder. Conversely, even in the case of monozygotic twins who share $100 \%$ of their genetic makeup, the concordance for psychiatric disorders rarely exceeds $50 \%$. This, of course, brings the whole issue of nongenetic contribution to the discussion, which, in most psychiatric disorders, was demonstrated to be nonshared in nature (as opposed to shared environmental risk factors within families). Analyzing these nonshared factors might be many orders of magnitude more complex than the genetic factors, given that our access to environmental factors is retrospective and tainted by various biases and circular causality. It is also quite possible that the nonshared environmental factors include all the "decisions" that an individual makes across every juncture in their life, and not only the physical environment to which a person is subjected. In that sense, the nonshared environment could also be of our own making. Eric Turkheimer took this idea to its extreme and made a bold and provocative proposition that the nonshared environment is free will:26

(...) Not the kind of metaphysical free will that no one believes in anymore, according to which human souls float free above the mechanistic constraints of the physical world, but an embodied free will, tethered to biology, that encompasses our ability to respond to complex circumstances in complex and unpredictable ways and in the process to build a self.

It can be argued that these conclusions may be specific to genetics, and other lines of biological investigations (laboratory study in the Robins and Guze proposal) may lead us to different, better delineations of specific psychiatric disorders. Notwithstanding the fact that most research in other biological fields of psychiatry remains impeded by small sample sizes and lack of robustness, some evidence suggests that the level of heterogeneity in psychiatric disorders is such that validation of specific mental disorders on the basis of brain imaging markers might be very difficult. For example, in a recent large study comparing brain structures in patients with schizophrenia $(n=163)$, bipolar disorder $(n=190)$ and controls $(n=256)$, it was concluded that the group-level differences disguised a high level of heterogeneity within disorders. Indeed, overlap of more than $2 \%$ among patients was reported only in a few loci, suggesting that sharing a major DSM diagnosis does not guarantee a meaningful commonality at the brain structure level. ${ }^{27}$ 


\section{A new mechanical philosophy of science}

From the philosophy of enlightenment ${ }^{28}$ to the logical empiricism $^{29}$ that dominated the epistemology of science until the late 20th century, important efforts have been made to understand phenomena on the basis of empirical evidence aided by logic and mathematics, with the ultimate goal of identifying the laws of nature on which we can base our understanding of phenomena. Much of the work that has been done by the classical writers in psychiatry had that same aspiration. At the turn of the 21st century, a new mechanical philosophy of science (NMP) emerged mainly from philosophical reflections on the foundations of biological sciences, particularly neuroscience and psychology. The main impetus for the NMP was the absence of general laws in biological sciences compared with physics and chemistry. The concept of mechanism is central to this NMP and was first articulated clearly in a paper by Machamer and colleagues published in 2000. ${ }^{30}$ In this seminal paper, it was proposed that "mechanisms are sought to explain how a phenomenon comes about or how some significant process works. Specifically: mechanisms are entities and activities organized such that they are productive of regular changes from start or set-up to finish or termination conditions." In this NMP, mechanisms are defined in a very large sense, and they can span multiple levels of analyses. They are not necessarily determinist, reductionist, sequential/linear or localizable; nor are they limited to "cogwheel" kinds of Cartesian machines. In addition, mechanisms are not laws of nature. ${ }^{31}$

In psychiatry, Kenneth Kendler ${ }^{32}$ and many others have promoted this new mechanistic approach to our field. In a paper titled "Explanatory models for psychiatric disorders," Kendler wrote: ${ }^{33}$

A more appropriate scientific model for psychiatry emphasizes the understanding of mechanisms, an approach that fits naturally with a multicausal framework and provides a realistic paradigm for scientific progress; that is, understanding mechanisms through decomposition and reassembly. (...) Biology will implement but not replace psychology within our explanatory systems.

Whether this new mechanistic approach will fill the gap between explanation (based on pure biological underpinnings) and understanding (based on psychological/phenomenological relatedness to patients) of mental phenomena, as formulated by Jaspers, ${ }^{34}$ the founder of phenomenological psychiatry, and later philosophers of science, ${ }^{35}$ remains an open question, although in a more recent paper Kendler and Campbell ${ }^{36}$ proposed some examples on how understanding of the life experience of patients at the phenomenological level can be enhanced by mechanistic explanations at the molecular and cognitive levels.

\section{Conclusion}

Importantly, we are not presenting a negative view of the NKF or trying to denigrate what it has achieved; on the contrary, we strongly believe that NKF has been extremely important for the development of psychiatric science in the last 50 years and that it definitely established that mental dis- orders are a reality etched in our genes and bodies, and not only social constructs. Genetic and brain imaging research have been the flagship of scientific validation of this framework, and their results clearly indicate a broad validation of psychiatric disorders as a group, but still with limited specificity and very few implications at the individual level. We believe that deeper phenomenological analyses of patients' experiences are needed and should be taught, researched and cherished. This will be very useful from a clinical point of view (at the individual level) and will open new vistas of research on psychological/phenomenological mechanisms, fully rehabilitated within the new mechanistic philosophy of science, and not as epiphenomena that can be disposed of as soon as they are correlated to whatever molecular or brain activity phenomenon. In that, the NKF will have contributed to the return of common sense.

Affiliations: From the Research Centre, Douglas University Institute, Montreal, Que., Canada; and the Department of Psychiatry, McGill University, Montreal, Que., Canada.

Competing interests: R. Joober reports grants, speaker fees and consultant honoraria from Janssen, Lundbeck, Otsuka, Pfizer, Shire, Purdue, HLS and Myelin, and royalties from Henry Stewart Talks, all unrelated to this work. No other competing interests declared.

\section{References}

1. Andreasen NC. DSM and the death of phenomenology in America: an example of unintended consequences. Schizophr Bull 2007;33:108-12.

2. Kuhn T. The Structure of Scientific Revolutions, 2 ed. Chicago: University of Chicago Press 19701970.

3. Kendler KS, Jablensky A. Kraepelin's concept of psychiatric illness. Psychol Med 2011;41:1119-26.

4. Kendler KS, Engstrom EJ. Kahlbaum, Hecker, and Kraepelin and the transition from psychiatric symptom complexes to empirical disease forms. Am J Psychiatry 2017;174:102-9.

5. Kendler KS. The transformation of American psychiatric nosology at the dawn of the twentieth century. Mol Psychiatry 2016;21:152-8.

6. Grünbaum A. Is Freudian psychoanalytic theory pseudoscientific by Karl Popper's criterion of demarcation? Am Philos $Q$ 1979;16:131-41.

7. Kandel ER. A new intellectual framework for psychiatry. Am J Psychiatry 1998;155:457-69.

8. Kandel ER. Biology and the future of psychoanalysis: a new intellectual framework for psychiatry revisited. Am J Psychiatry 1999;156:505-24.

9. Kendell RE, Cooper JE, Gourlay AJ, et al. Diagnostic criteria of American and British psychiatrists. Arch Gen Psychiatry 1971;25: 123-30.

10. Kendell RE. Psychiatric diagnosis in Britain and the United States. Br J Psychiatry 1975; (Spec No 9):453-61.

11. Tatossian A. A Fenomenologia das Psicoses. São Paulo: Escuta; 2016.

12. Tamelini MG, Messas GP. On the phenomenology of delusion: the revelation of its aprioristic structures and the consequences for clinical practice. Psicopatologia Fenomenológica Contemporânea. 2016;5:1-21.

13. Bortolotti L. Delusions and other irrational beliefs. Oxford: Oxford university Press; 2010.

14. Robins E, Guze SB. Establishment of diagnostic validity in psychiatric illness: its application to schizophrenia. Am J Psychiatry 1970;126:983-7. 
15. Sherrington R, Brynjolfsson J, Petursson H, et al. Localization of a susceptibility locus for schizophrenia on chromosome 5. Nature 1988;336:164-7.

16. Detera-Wadleigh SD, Berrettini WH, Goldin LR, et al. Close linkage of c-Harvey-ras-1 and the insulin gene to affective disorder is ruled out in three North American pedigrees. Nature 1987;325:806-8.

17. $\mathrm{Li} \mathrm{Z}$, Chen J, Yu H, et al. Genome-wide association analysis identifies 30 new susceptibility loci for schizophrenia. Nat Genet 2017;49:1576-83.

18. Cross-Disorder Group of the Psychiatric Genomics, Lee SH, Ripke $\mathrm{S}$, et al. Genetic relationship between five psychiatric disorders estimated from genome-wide SNPs. Nat Genet 2013;45:984-94.

19. Witt SH, Streit F, Jungkunz M, et al. Genome-wide association study of borderline personality disorder reveals genetic overlap with bipolar disorder, major depression and schizophrenia. Transl Psychiatry 2017;7:e1155.

20. Autism Spectrum Disorders Working Group of The Psychiatric Genomics. Meta-analysis of GWAS of over 16,000 individuals with autism spectrum disorder highlights a novel locus at 10q24.32 and a significant overlap with schizophrenia. Mol Autism 2017;8:21.

21. Savage JE, Jansen PR, Stringer S, et al. Genome-wide association meta-analysis in 269,867 individuals identifies new genetic and functional links to intelligence. Nat Genet 2018;50:912-9.

22. Brainstorm Consortium, Anttila V, Bulik-Sullivan B, et al. Analysis of shared heritability in common disorders of the brain. Science 2018;360: pii: eaap8757.

23. Kirov G, Rees E, Walters JT, et al. The penetrance of copy number variations for schizophrenia and developmental delay. Biol Psychiatry 2014;75:378-85.

24. Loh PR, Bhatia G, Gusev A, et al. Contrasting genetic architectures of schizophrenia and other complex diseases using fast variancecomponents analysis. Nat Genet 2015;47:1385-92.

25. Boyle EA, Li YI, Pritchard JK. An expanded view of complex traits: from polygenic to omnigenic. Cell 2017;169:1177-86

26. Turkheimer E. Genetics and human agency: comment on DarNimrod and Heine (2011). Psychol Bull 2011;137:825-8.

27. Wolfers T, Doan NT, Kaufmann T, et al. Mapping the heterogeneous phenotype of schizophrenia and bipolar disorder using normative models. JAMA Psychiatry 2018;75:1146-55.

28. Bristow W. Enlightenment. Stanford Encyclopedia of Philosophy Archive; 2017. Available: https://plato.stanford.edu/archives/ fall2017/entries/enlightenment/(accessed 2018 Dec. 6).

29. Creath R. Logical empiricis. Stanford Encyclopedia of Philosophy Archive; 2017. Available: https://plato.stanford.edu/archives / fall2017/entries/logical-empiricism (accessed 2018 Dec. 6).

30. Machamer P, Darden L, Craver CF. Thinking about mechanisms. Philos Sci 2000;67:1-27.

31. Craver C, Tabery J. Mechanisms in science. Stanford Encyclopedia of Philosophy; 2017. Available: https:/ / plato.stanford.edu/archives / spr2017/entries/science-mechanisms/ (accessed 2018 Dec. 6).

32. Kendler KS, Zachar P, Craver C. What kinds of things are psychiatric disorders? Psychol Med 2011;41:1143-50.

33. Kendler KS. Explanatory models for psychiatric illness. Am J Psychiatry 2008;165:695-702.

34. Doerr-Zegers $\mathrm{O}$, Peligrina-Cetràn H. Jarl Jaspers' general psychopathology in the framework of clinical practice. In: Stanghellini G, Fuchs M, editors. One Century of Karl Japers' general psychopathology. Oxford: Oxford University Press; 2013.

35. Levine J. Materialism and qualia: the explanatory gap. Pacific Philosophical Quarterly. 1983;64:354-61.

36. Kendler KS, Campbell J. Expanding the domain of the understandable in psychiatric illness: an updating of the Jasperian framework of explanation and understanding. Psychol Med 2014;44:1-7.

\section{JPN's top viewed articles*}

1. Why is depression more prevalent in women? Albert

J Psychiatry Neurosci 2015;40(4):219-21

2. N-acetylcysteine in psychiatry: current therapeutic evidence and potential mechanisms of action Dean et al.

J Psychiatry Neurosci 2011;36(2):78-86

3. Mental illness is like any other illness: a critical examination of the statement and its impact on patient care and society

Malla et al.

J Psychiatry Neurosci 2015;40(3):147-50

4. Legalizing marijuana

Leyton

J Psychiatry Neurosci 2016;41(2):75-6

5. Possible role of more positive social behaviour in the clinical effect of antidepressant drugs

Young et al.

J Psychiatry Neurosci 2014;39(1):60-5

6. Mental wellness in Canada's Aboriginal communities: striving toward reconcilliation Boksa et al.

J Psychiatry Neurosci 2015;40(6):363-5

7. Inappropriate benzodiazepine use in elderly patients and its reduction

Tannenbaum

J Psychiatry Neurosci 2015;40(3):E27-8

8. On the neurobiology of hallucinations

Boksa

J Psychiatry Neurosci 2009;34(4):260-2

9. Mindfullness-based treatments for posttraumatic stress disorder: a review of the treatment literature and neurobiological evidence

Boyd et al.

J Psychiatry Neurosci 2018;43(1):7-25

10. Publication bias: What are the challenges and can they be overcome?

Joober et al.

J Psychiatry Neurosci 2012;37(3):149-52

*Based on page views on PubMed Central of content published in the last 10 years.

Updated Dec. 10, 2018 